\title{
The Effect of Recent Jet Results on MSTW PDFs
}

\section{Benjamin J.A. Watt}

University College London

E-mail: benjamin.watt.09@ucl.ac.uk

\begin{abstract}
The effect of recent ATLAS inclusive- and di-jet results on the current standard PDF sets is investigated, and compared and contrasted to similar results from the Tevatron. We investigate the effect of the data using the parton reweighting technique. For ATLAS inclusive jets, there is good agreement across all values of $\mathrm{x}$, and no significant pull is seen on the gluon. For dijets, both D0 and ATLAS data have a potential impact on the PDFs, and the optimum gluon is not necessarily in agreement with that required by the commensurate inclusive data, however the form of the theoretical prediction must be closely investigated, with significant sensitivity to scale choice observed.
\end{abstract}

XXI International Workshop on Deep-Inelastic Scattering and Related Subject -DIS2013, 22-26 April 2013

Marseilles, France

${ }^{*}$ Speaker. 


\section{ATLAS Inclusive Jets}

Since the last update of the PDFs from the MSTW group [1], the LHC has begun operations and produced data which will have an impact on the knowledge of proton structure. The $\left(x, Q^{2}\right)$ phasespace probed at the LHC covers a much larger region than the data used in the current PDF fits, and so using this early LHC data will provide information on the effectiveness of the DGLAP model of parton evolution.

The data used in this study are the ATLAS inclusive jet and dijet cross sections at $7 \mathrm{TeV}$ centre of mass energy [2]. Dijet data of any kind is yet to be included in the MSTW fits, and so a study is performed into the feasability of using such data by comparing the stability of calculations at the Tevatron and LHC.

The calculations for both inclusive and dijet cross sections are performed at next to leading order using the NLOjet++ [3] [4] program, interfaced with APPLgrid [5] for apostiori scale variations and PDF eigenvector changes. The ratio of the inclusive jet cross section data to the calculation is shown in Fig 1. The red points show the ratio before considering experimental systematic errors, and the fit is clearly poor with all data points above the theory. The picture is not complete, however, until the systematic uncertainties are properly accounted for. In this

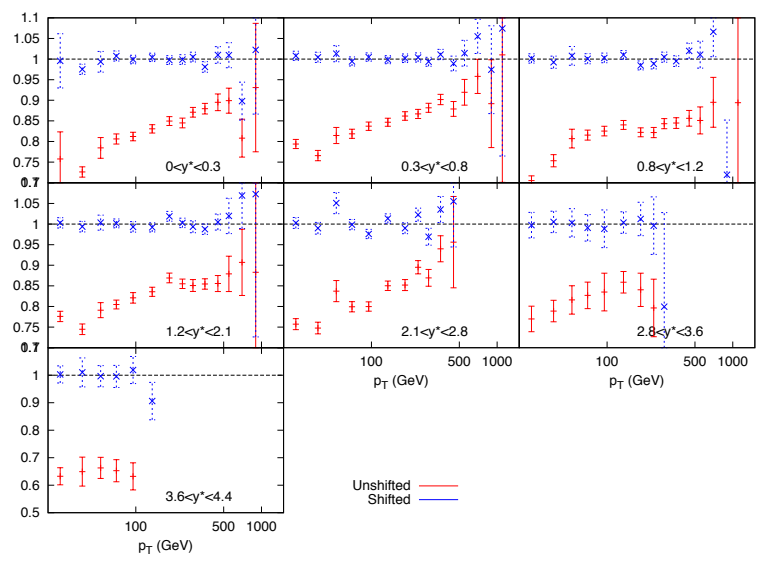

Figure 1: Ratio of data to theory for ATLAS inclusive jets data set, there are 88 sources of correlated systematic uncertainty, all of which can cause the effected data points to move up or down. The blue points show the data/theory ratio after these effects are considered. In order to include this in a fit, the following $\chi^{2}$ goodness of fit formula is used:

$$
\chi^{2}=\sum_{i=1}^{N_{\text {pts }}}\left(\frac{D_{i}-\sum_{k=1}^{N_{\text {corr }}} r_{k} \sigma_{k, i}^{\text {corr }}-T_{i}}{\sigma_{i}^{\text {uncorr }}}\right)^{2}+\sum_{k=1}^{N_{\text {corr }}} r_{k}^{2}
$$

where $D_{i}, T_{i}$ are the data and theory points, $\sigma_{i}^{\text {uncorr }}$ is the sum in quadrature of all sources of uncorrelated uncertainty, and $r_{k}$ are the nuisance parameters introduced to allow the systematics to move the data points. The results of the fit for the MSTW2008 NLO PDF set is shown in Table 1, along with the distribution of $r_{k}$ parameters in Table 2 .

\begin{tabular}{cccc}
\hline \hline Scale & $\mathrm{pT} / 2$ & $\mathrm{pT}$ & $2 \mathrm{pT}$ \\
\hline $\mathrm{R}=0.4$ & 0.75 & 0.78 & 0.70 \\
$\mathrm{R}=0.6$ & 0.85 & 0.79 & 0.72 \\
\hline
\end{tabular}

Table 1: $\chi^{2}$ per point $(90$ points) reweighting procedure introduced in [6] and more specifically for MSTW in [7] is used. Firsty, the prediction for each eigenvector in the MSTW2008 fit is produced. These predictions are combined to produce $1000 \mathrm{PDFs}$ randomly distributed in eigenvector space, using the formula: 


$$
F\left(S_{k}\right)=F\left(S_{0}\right)+\sum_{j=1}^{n}\left[F\left(S_{j}^{ \pm}\right)-F\left(S_{0}\right)\right]\left|R_{j k}\right|
$$

\begin{tabular}{ccccc}
\hline \hline$\left|r_{k}\right|<$ & 0.5 & 1.5 & 2.5 & 3.5 \\
\hline $\mathrm{R}=0.4$ & 72 & 15 & 1 & 0 \\
$\mathrm{R}=0.6$ & 74 & 13 & 1 & 0 \\
\hline
\end{tabular}

Table 2: Distribution of $r_{k} \mathrm{~s}$ (Total 88)

Each random PDF is weighed according to its $\chi^{2}$, and by statistical combination can provide an updated ideal PDF for the dataset in question.

For the ATLAS inclusive jet cross section, the reweighted gluon PDFs are shown in Fig 2. There is minimal change in both the central value and error bands of the PDFs. Also, the effective number of PDFs used in the reweighted PDF, $N_{\text {eff }}$, is very large for both $\mathrm{R}$ parameters. This is a measure of the effect of the data on the PDFs, with more effective PDFs implying the reweighting has little effect. Clearly the MSTW2008 PDFs describe this data excellently, but the large systematic errors are the main cause for this quality of fit, and so more discerning data is required.

\section{D0 and ATLAS Dijets}

When considering dijet production, the choice of renormalisation and factorisation scales to include in the NLO calculation is not obvious. Unlike inclusive jet production, in which the only physical scale involved
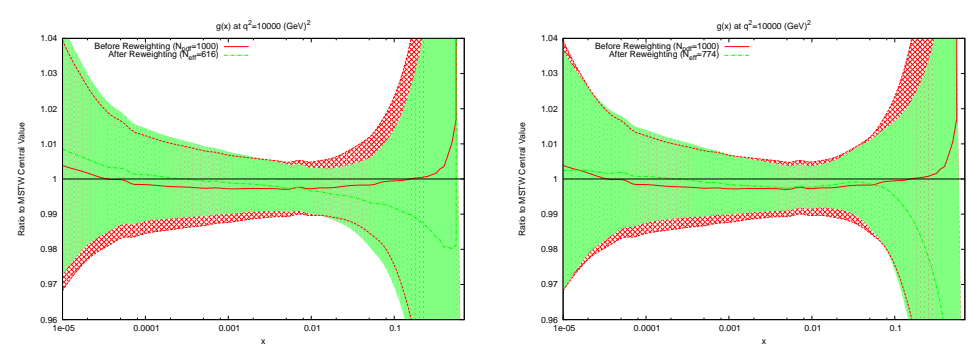

Figure 2: The effect of reweighting the MSTW2008 gluon using ATLAS inclusive jet data. Jet size parameter $R=0.4$ (left), and $R=0.6$ (right).

in the events is the $p_{T}$ of the jet, dijet production has a number of possible choices of scale. The seemingly most obvious choice is the average $p_{T}$ of the two jets, however at high rapidities this can lead to problems due to the possible configuration of the event. A highly boosted hard scatter will have the same average $p_{T}$ as an unboosted soft scatter. Another variable which could be used as the scale choice is the dijet mass, $M_{J J}$, which would not suffer from the issues in event classification at high rapidities. Fig 3 demonstrates the benefit of using dijet mass as the scale choice. In the case of $p_{T}^{a v}$, although at low rapidity the prediction is stable and flat across all $M_{J J}$, the predictions from different multiplicative factors of the scale begin to cross in the more forward bins. This has already been observed in [8], however other scale choices were not investigated.

In comparison, the theory/data ratio for the $M_{J J}$

\begin{tabular}{cccc}
\hline \hline Multiplier & 0.5 & 1.0 & 2.0 \\
\hline$p_{T}^{a v}$ & 3.23 & 2.34 & 1.61 \\
$M_{J J}$ & 1.88 & 1.29 & 1.06 \\
$\frac{M_{J J}}{2 \cosh (0.7 y *)}$ & 3.06 & 2.15 & 1.44 \\
\hline
\end{tabular}

Table 3: $\chi^{2}$ values for D0 dijets calculation is much more stable. The variation through multiplicative factors of the scale are constant throughout all rapidity bins, and the ratio remains generally flat. The $\chi^{2}$ values shown in Table 3 confirms that the choice of $M_{J J}$ provides the better fit. Also calculated is another choice of scale, namely multiples of $\frac{M_{J J}}{2 \cosh (0.7 y *)}$ suggested in [9], to allow more dependance on the dijet rapidity to be probed. This scale choice, while also an improvement on the $p_{T}^{a v}$ calculation, does not provide a better fit than using simply $M_{J J}$ alone. 

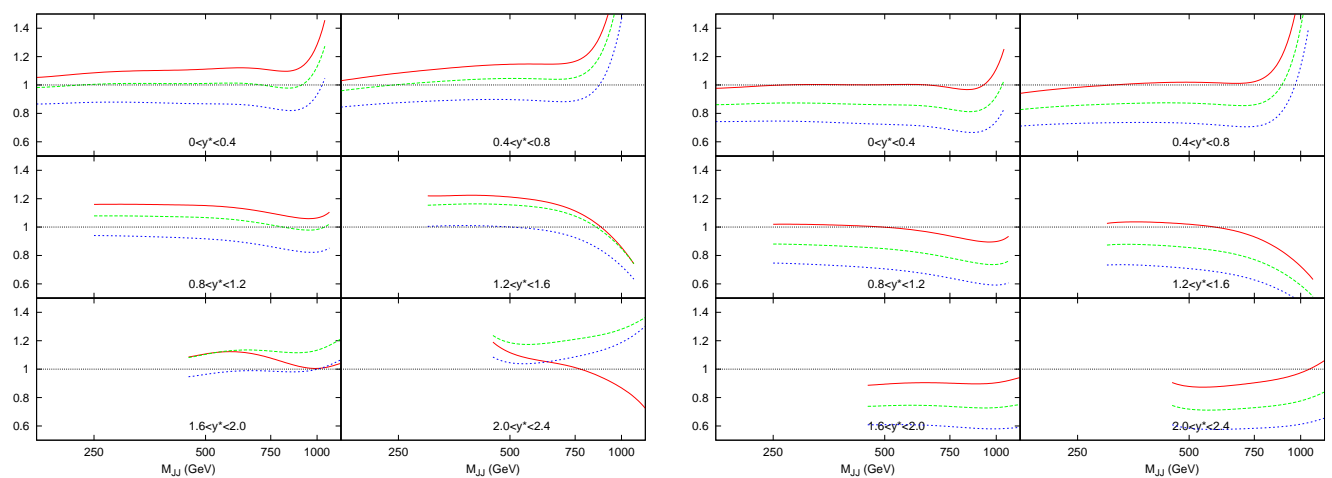

Figure 3: Theory/Data ratio for D0 dijets, using multiples of $p_{T}^{a v}$ (left) and $M_{J J}$ (right) as the choice of $\mu_{R}$ and $\mu_{F}$. The multiples are 0.5 (red), 1.0 (green) \& 2.0 (blue)

Fig 4 demonstrates the scale variation of two single points in the kinematic phasespace. Both are in the lowest $y^{*}$ bin, however the first includes dijets with low mass $(70-110 \mathrm{GeV})$ and the second includes those with high mass (1940-2780 GeV). The general behaviour is that of a stable saddle region in the central region, with data/theory decreasing away from the saddle in along one axis and increasing along the other. The axes defining the saddle region, however, differ greatly between the two points. A smooth rotation anticlockwise is observed as the dijet mass is increased, resulting in the large rotation shown in the figure. This behaviour was first noted in other cross sections in [10].

The dependence of this rotation on the kinematic variables is shown more clearly in Fig 5, where only the rapidity bin is changed. The $1.18 \mathrm{TeV}<M_{J J}<1.31 \mathrm{TeV}$ bin is chosen for study as this is the bin appearing in the most rapidity bins. It is clear that the angle of the saddle point is dependant only on
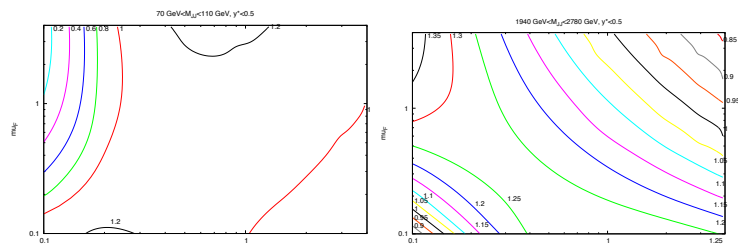

Figure 4: Scale variations of two points with the same rapidity but differing $M_{J J}$, using $M_{J J}$ as the kinematical scale choice. the dijet mass, however the overall behaviour is still affected by the rapidity. A migration towards lower scale choices is seen, such that at the highest rapidities, the saddle point disappears and the surface simply becomes a unidirectional slope.

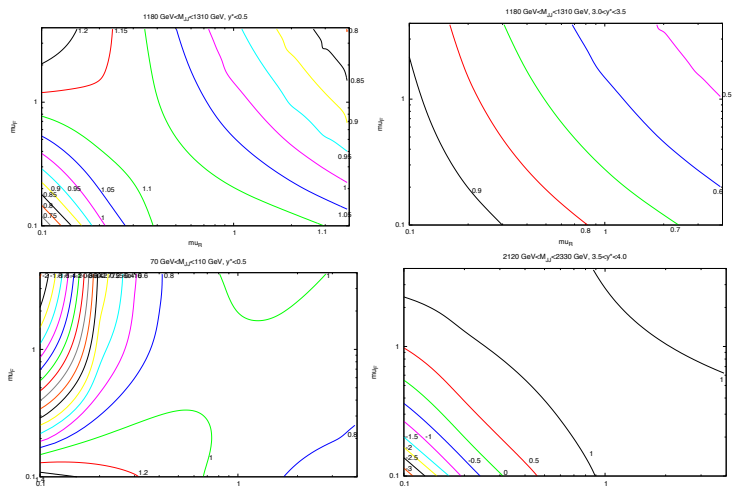

Figure 5: Scale variations of two points with the same $M_{J J}$ but differing rapidity, using $M_{J J}$ as the kinematical scale choice.

results for ATLAS dijets are shown in Table 4. Here, the best fit is obtained for a large multi-
Ideally, the scale choice for a calculamost stable calculation, and hence would be within the saddle region for all of the points in the dataset. Finally the effects on the PDFs themselves are studied. The same reweighting procedure is used as for the inclusive jets, and the effect for each scale choice for both D0 and ATLAS dijets is shown in Fig 6. The D0 dijet data clearly has a significant effect on the gluon, despite the commensurate inclusive data being included in the fit. The fit
re, the best fit is obtained for a large multition would be the one which provides the 
plier of $p_{T}^{a v}$, although this calculation breaks down for small multipliers. $M_{J J}$ and $\frac{M_{J J}}{\cosh (0.7 y)}$ both do reasonably well for all multipliers.

\begin{tabular}{cccc}
\hline \hline Multiplier & 0.5 & 1.0 & 2.0 \\
\hline $2 * p_{T}^{a v}$ & 6.66 & 1.95 & 1.90 \\
$M_{J J}$ & 2.05 & 2.41 & 2.98 \\
$\frac{M_{J J}}{\cosh (0.7 y)}$ & 2.59 & 2.27 & 2.12 \\
\hline
\end{tabular}

Table 4: Table of $\chi^{2}$ values (138 points) for ATLAS dijet with different scale choices.
There is however a difference in the effect depending on the scale choice used. For ATLAS dijets, a similar effect is seen for the preferred gluon, but a larger dependance is observed on the scale choice, with $M_{J J}$ requiring a very different shape. Including dijet data in PDF fits will, then, have a noticable effect, but further study into the scale uncertainties from the choice and theoretical uncertainties in general is required.
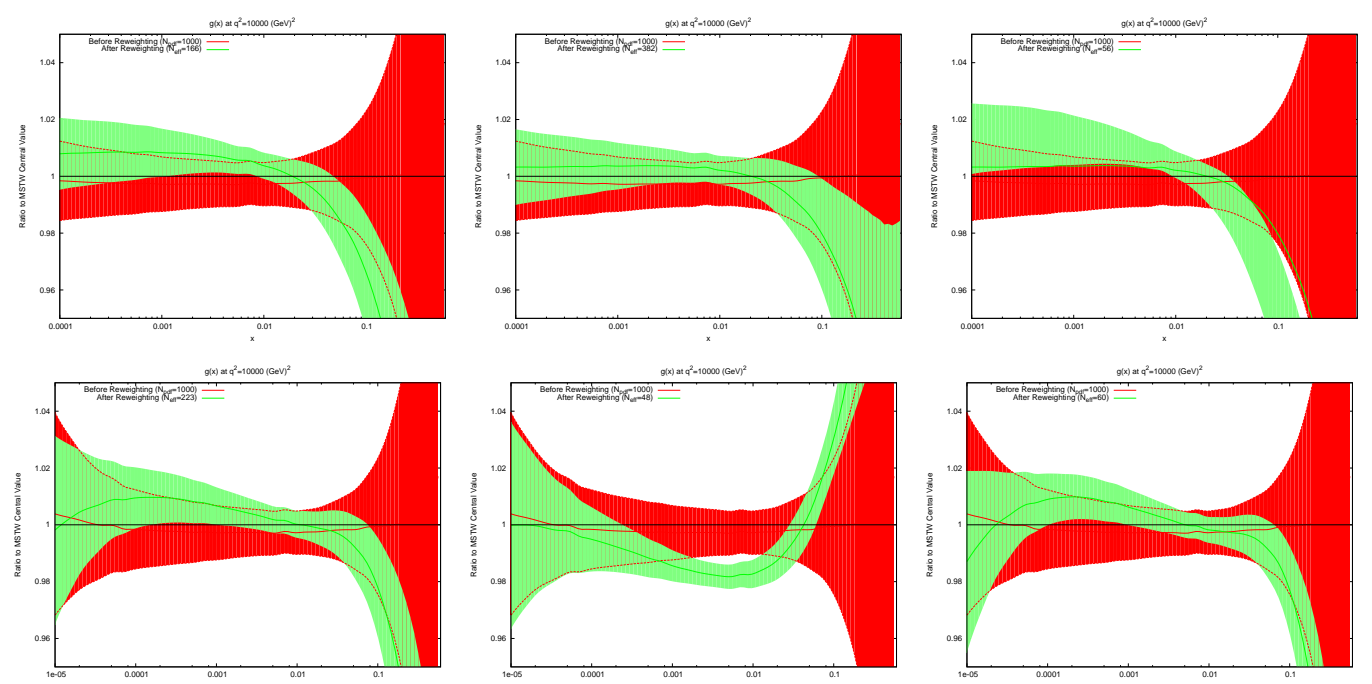

Figure 6: The effect of PDF reweighting on the gluon for 3 different scale choices for D0 (top) and ATLAS (bottom) dijets. From left to right, the scale choices are: $p_{T}^{a v}, M_{J J}$, and $M_{J J} / 0.7 \cosh \left(y^{*}\right)$

\section{References}

[1] A. D. Martin et al, Eur.Phys.J. C63 (2009) 189Đ285, [arXiv:0901.0002]

[2] ATLAS Collaboration,Phys.Rev. D86 (2012) 014022, [arXiv:1112.6297]

[3] Z.Nagy Phys. Rev. D68, 094002 (2003)

[4] Z. Nagy, Phys. Rev. Lett.88, 122003 (2002)

[5] T. Carli et al, Eur Phys J C 66 (2010) 503, [arXiv:0911.2985]

[6] NNPDF Collaboration, Nucl.Phys.B855:608-638,2012,[arXiv:1108.1758]

[7] G. Watt and R. S. Thorne,JHEP 08 (2012) 052 [arXiv:1205.4024]

[8] D0 Collaboration, Phys.Lett.B693:531-538,2010, [arXiv:1002.4594]

[9] S. D.Ellis, et al, Phys. Rev. Lett. 69, 1496 (1992)

[10] Talk by J.Huston - https://indico.cern.ch/contributionDisplay.py?contribId=3\&confId=226756 